\title{
Stochastic agent-based modeling of tuberculosis in Canadian Indigenous communities
}

\author{
Ashleigh R. Tuite ${ }^{1,5^{*}}$, Victor Gallant ${ }^{2}$, Elaine Randell ${ }^{3}$, Annie-Claude Bourgeois ${ }^{2}$ and Amy L. Greer ${ }^{4}$
}

\begin{abstract}
Background: In Canada, active tuberculosis (TB) disease rates remain disproportionately higher among the Indigenous population, especially among the Inuit in the north. We used mathematical modeling to evaluate how interventions might enhance existing TB control efforts in a region of Nunavut.

Methods: We developed a stochastic, agent-based model of TB transmission that captured the unique household and community structure. Evaluated interventions included: (i) rapid treatment of active cases; (ii) rapid contact tracing; (iii) expanded screening programs for latent TB infection (LTBI); and (iv) reduced household density. The outcomes of interest were incident TB infections and total diagnosed active TB disease over a 10- year time period.

Results: Model-projected incidence in the absence of additional interventions was highly variable (range: 33-369 cases) over 10 years. Compared to the 'no additional intervention' scenario, reducing the time between onset of active TB disease and initiation of treatment reduced both the number of new TB infections (47\% reduction, relative risk of $\mathrm{TB}=0.53)$ and diagnoses of active $\mathrm{TB}$ disease $(19 \%$ reduction, relative risk of $\mathrm{TB}=0.81)$. Expanding general population screening was also projected to reduce the burden of $\mathrm{TB}$, although these findings were sensitive to assumptions around the relative amount of transmission occurring outside of households. Other potential interventions examined in the model (school-based screening, rapid contact tracing, and reduced household density) were found to have limited effectiveness.
\end{abstract}

Conclusions: In a region of northern Canada experiencing a significant TB burden, more rapid treatment initiation in active TB cases was the most impactful intervention evaluated. Mathematical modeling can provide guidance for allocation of limited resources in a way that minimizes disease transmission and protects population health.

Keywords: Canada, Latent TB infection, Mathematical model, Nunavut, Public health, Simulation, TB, Tuberculosis

\section{Background}

Tuberculosis (TB) is an ongoing public health issue with Canadian-born Indigenous peoples disproportionately affected. Between 1970 and 2010, the proportion of active TB cases in Canadian-born Indigenous peoples increased from 14.7 to $21.2 \%$ [1]. Indigenous communities experience higher rates of active $\mathrm{TB}$ disease than

\footnotetext{
* Correspondence: atuite@hsph.harvard.edu

'Dalla Lana School of Public Health, University of Toronto, University of Toronto, Toronto, ON, Canada

${ }^{5}$ Harvard T.H. Chan School of Public Health, 1633 Tremont Street, Boston, MA 02120, United States

Full list of author information is available at the end of the article
}

Canadian non-Indigenous populations. Determinants of TB infection and disease differ between Canadian Indigenous peoples and Canadian non-Indigenous populations [2]. Canadian Indigenous peoples experience significant differences in terms of comorbidities, transmission factors, and social determinants of health, compared to the non-Indigenous population [2].

In the Canadian territory of Nunavut specifically, there were 581 TB cases reported to the Nunavut Department of Health in a 10-year period between 1999 and 2011 [3]. Almost all reported cases between 1999 and 2011 (98.8\%) were of Inuit origin [3]. In 2010, the highest 
annual number of cases was reported in Nunavut with 100 active cases (304.7 cases per 100,000 population) identified [4]. In 2012, the TB incidence rate in Nunavut (which is home to $49 \%$ of the total Inuit population in Canada) was 234 cases per 100,000 population, almost 50 times the overall Canadian rate (4.8 per 100,000) [4]. In Nunavut, over $90 \%$ of identified active TB cases between 1999 and 2011 received treatment with adherence rates of $80 \%$ or better [3]. Public health awareness campaigns have demonstrated success in increasing TB testing rates within Iqaluit, the capital of Nunavut. However, this level of testing was not sustained after the completion of the awareness campaigns [5]. The First Nations and Inuit Health Branch (FNIHB) of the Government of Canada set a goal of reducing TB in the Inuit population of Canada to 3.6 cases per 100,000 population by 2015 [2]. Recent data indicate that this goal has not been met, suggesting that additional public health strategies in addition to routine contact tracing and screening are necessary to address TB in Nunavut [5].

The prevalence of socioeconomic factors that contribute to infection and disease is a particular challenge to controlling TB in Nunavut $[6,7]$. Overcrowded housing with poor ventilation is common [8], and may facilitate the transmission of $\mathrm{TB}$ and other airborne respiratory infections [7]. There have been calls for additional resources for increased access to diagnostic testing, treatment, contact tracing, and more comprehensive screening programs, as well as more long-term investments to address housing, poverty, and food security [7].

While TB shares many attributes with other communicable diseases, it is distinguished by the high frequency with which latent infection occurs, and by the tendency of a small percentage of latent infections to reactivate and progress to active TB disease years or decades after initial infection [9]. This complicates the control of TB, as high TB rates in communities are likely be due to a combination of recent transmission events and reactivation of infection in latently infected individuals.

Given the unique characteristics of $\mathrm{TB}$, including potentially long lags between infection and disease onset and the challenges of conducting sufficiently powered trials [10], disease transmission models are frequently used to evaluate the impact of TB control policy options [11-15]. These models facilitate the evaluation of different strategies that might impact TB control, such that we can gain a better idea of how best to allocate limited resources in a way that minimizes disease transmission and protects the health of at-risk populations.

We sought to develop a stochastic, agent-based simulation model to describe TB transmission and evaluate different intervention strategies that might be used to control the spread of TB in Canada's north. The model focused on the Kivalliq Region of Nunavut, which encompasses seven communities and is an area that continues to experience a high burden of TB [16].

\section{Methods \\ Model overview}

We developed an agent-based simulation model of Mycobacterium tuberculosis transmission in the Kivalliq Region of Nunavut, Canada (Fig. 1). The region has a population of 8952 residents and is home to seven distinct communities ranging in size from 310 to 2320 individuals [3]. This model represents individuals (agents) within a simulated environment, and their interactions, movements, decision-making, and related health states. We used an agent-based approach to account for the small population size and associated stochasticity. This approach allowed us to model the unique household and community structure in this region, and to record the health states and treatment histories of individuals over time. The model was constructed using the AnyLogic software package (http://www.anylogic.com/). Model parameters were region-specific, wherever possible, or derived from the biomedical literature (Table 1).

A brief summary of the model structure and calibration procedure is provided below, with a more complete description included in the Additional file 1.

\section{Population and community structure}

To reflect the demographic structure of Kivalliq, individuals in the model were assigned an age, sex, household, and community. The initial age distribution of the population was based on 2001 Canadian census estimates for the Kivalliq Region [17]. Each individual was assigned to a household, which in turn was located within one of the seven communities. Average household size was based on census data [18, 19]. New households were added every year. Individuals were added to the model population by birth and left the population by death, with rates based on Nunavut data (Table 1) [20, 21]. Although we allowed for movement between communities (described in Additional file 1), we did not model migration into or out of the region.

\section{Natural history of tuberculosis}

After the initial synthetic model population was created as described above (agents assigned specific, individual attributes including an age, sex, household size, community, and specific individual household members), agents within the synthetic population were assigned a health state based on the TB natural history component of the model (Fig. 2). This aspect of the model represented each individual's health state over time. We included the following stages of the natural history of TB: susceptible, latent TB infection (LTBI), active disease, and 


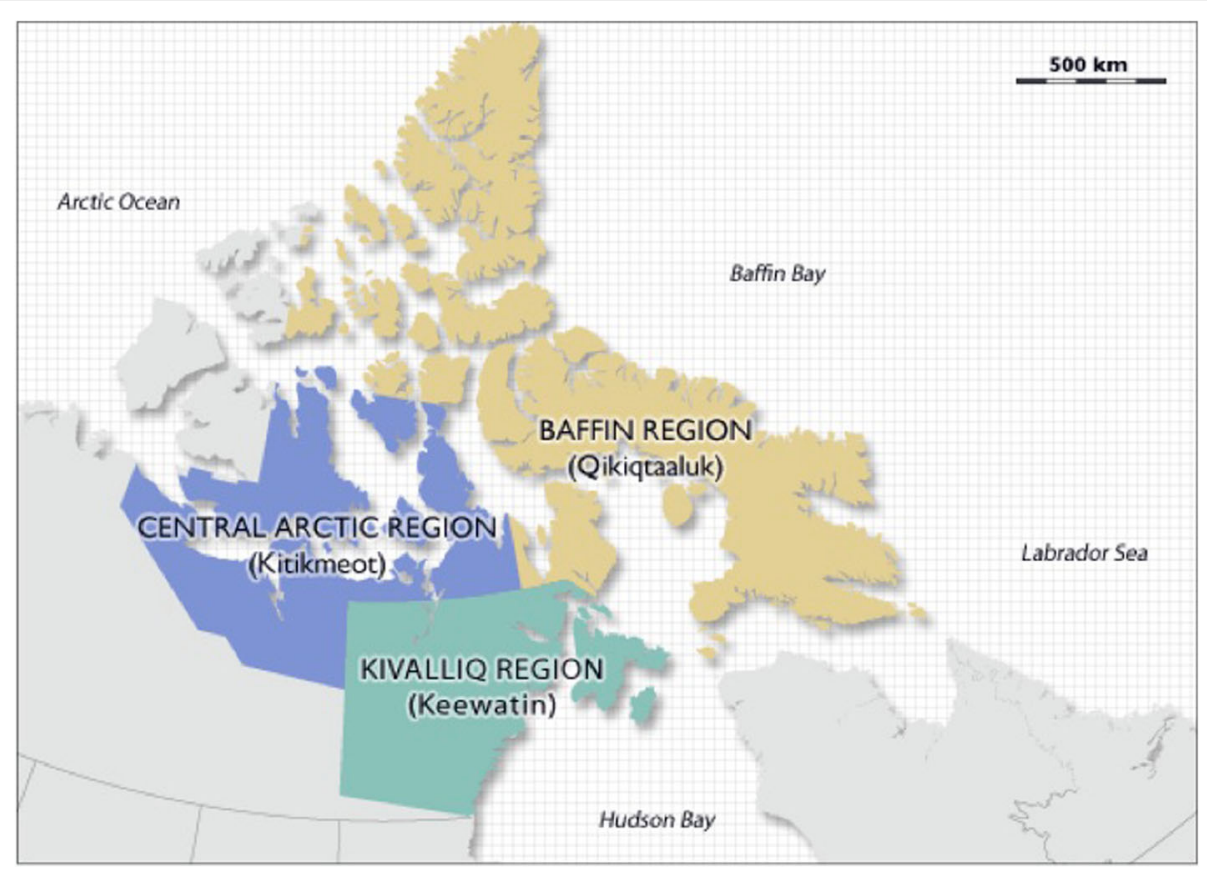

Fig. 1 Map showing the location of the Territory of Nunavut as well as the three distinct geographic regions. Kivalliq is the southernmost region and borders Hudson Bay

resusceptible. Susceptible individuals are TB naïve, having never been infected by TB before and can become infected if they come in contact with an individual with an active TB infection. Individuals who have been recently infected progress to the the latent TB classes (latent fast or latent slow). In this case, a small proportion of individuals will go on to develop active pulmonary TB within a period of 5 years (Table 1) with the remainder staying in the latent slow class where they can stay indefinitely or they can progress to the active TB state at some time in the future [22]. The active TB states are broken down into three distinct compartments: hightransmissibility, low transmissibility, and extrapulmonary active TB. Individuals in the high-transmissibility compartment are individuals who have smear-positive, pulmonary TB. These individuals are considered more infectious than individuals diagnosed with pulmonary TB but who are smear-negative (Table 1) [22]. We assume that individuals who are diagnosed with extrapulmonary TB are not infectious to others [22]. Individuals in either the LTBI states or the active TB states can transition into the 'diagnosed and treated' compartment based on parameters describing the rate of diagnosis and treatment of TB cases in Kivalliq, Nunavut (Table 1). Individuals in the model who have been successfully treated for TB or who spontaneously clear their TB without receiving treatment (Table 1), transition to the resusceptible compartment $[12,22]$. Individuals in the resusceptible compartment can become reinfected but their risk of acquiring a new $\mathrm{TB}$ infection is reduced compared to a TB naïve individual (Table 1) [12]. Parameters describing all model transitions between states are presented in Table 1 and are informed by both the existing biomedical literature and data extracted from the Nunavut TB registry. To capture age-related differences in TB infection, progression, and management, we classified individuals aged $<15$ years as 'children', and those aged $\geq 15$ as 'adults' based on the age cut-offs used in the Canadian Tuberculosis Standards ( $7^{\text {th }}$ edition) and the age groupings available from the Nunavut Department of Health [1, 22, 23]. We assumed that the majority of TB transmission occurred between individuals within a household (representing close contacts) [22]. However, we also included a community network (encompassing all agents living within an individual's community) to allow us to investigate the contribution of community and casual contacts to TB transmission.

\section{Contact tracing and latent tuberculosis infection screening}

We assumed that susceptible individuals, as well as individuals with LTBI or undiagnosed active disease who had no prior history of treatment for LTBI or active disease, could undergo screening. Those diagnosed with LTBI and aged between 6 months and 65 years could receive treatment, with a proportion of these individuals completing treatment [5, 24]. Those diagnosed with active disease received appropriate treatment. All 
Table 1 Model parameters, values, ranges, and sources

\begin{tabular}{|c|c|c|c|}
\hline Parameter & Details & Value & Source \\
\hline \multicolumn{4}{|l|}{ Tuberculosis Natural History Parameters } \\
\hline Probability of transmission (per contact) & & 0.1 & Abu-Raddad [12] \\
\hline Number of respiratory contacts (per year) & & $40-1000$ & Estimated by model calibration \\
\hline Proportion of transmission occurring in community & Varied & $0.01-0.15$ & Assumption \\
\hline \multirow{3}{*}{$\begin{array}{l}\text { Proportion of new infections entering latent fast state } \\
\text { (active disease in }<5 \text { years) }\end{array}$} & & & Abu-Raddad [12] \\
\hline & Adult & 0.15 & \\
\hline & Child & 0.05 & \\
\hline Progression to active disease (per year) & Fast progressor & 1.5 & Abu-Raddad [12] \\
\hline $\begin{array}{l}\text { Progression to active disease (lifetime probability, } \\
\text { rate dependent on age at infection) }\end{array}$ & Slow progressor & 0.05 & Abu-Raddad [12] \\
\hline \multirow[t]{3}{*}{ Proportion of active cases with extrapulmonary disease } & & & Kivalliq surveillance data \\
\hline & Adult & 0.11 & \\
\hline & Child & 0.042 & \\
\hline \multirow{3}{*}{$\begin{array}{l}\text { Proportion of active cases with high transmissibility } \\
\text { pulmonary disease }\end{array}$} & & & Kivalliq surveillance data \\
\hline & Adult & 0.3 & \\
\hline & Child & 0.043 & \\
\hline \multirow{3}{*}{$\begin{array}{l}\text { Proportion of active cases with low transmissibility } \\
\text { pulmonary disease }\end{array}$} & & & Kivalliq surveillance data \\
\hline & Adult & 0.59 & \\
\hline & Child & 0.915 & \\
\hline \multirow[t]{3}{*}{ Infectivity (relative to high transmissibility TB) } & & & Abu-Raddad [12] \\
\hline & Low transmissibility TB & 0.25 & \\
\hline & Extrapulmonary TB & 0 & \\
\hline Spontaneous recovery rate (per year) & & 0.1 & Abu-Raddad [12] \\
\hline Relative susceptibility to re-infection & Resusceptible individuals & 0.6 & Vynnycky [33] \\
\hline Probability of TB-attributable mortality with active disease & & 0.0094 & Kivalliq surveillance data \\
\hline \multicolumn{4}{|l|}{ Population and Community Characteristics } \\
\hline Number of communities & & 7 & Census [18] \\
\hline Initial number of households & & 1890 & Census [19] \\
\hline Average household size & & 4 & Census [18] \\
\hline Number of new households added (per year) & & 30 & Census [18] \\
\hline Proportion of population $<15$ years of age & & 0.35 & Census [18] \\
\hline Initial number of individuals diagnosed and on treatment & & 2 & Kivalliq surveillance data \\
\hline \multirow{4}{*}{$\begin{array}{l}\text { Initial number of individuals in different states } \\
\text { (remaining are susceptible) }\end{array}$} & & & Estimated by model calibration \\
\hline & Undiagnosed LTBI & $10-2000$ & \\
\hline & Undiagnosed active TB disease & $1-50$ & \\
\hline & $\begin{array}{l}\text { Resusceptible (following treatment } \\
\text { or spontaneous recovery) }\end{array}$ & $50-3000$ & \\
\hline Birth rate (per year) & Females aged $15-44$ & 0.1 & Nunavut Bureau of Statistics [21] \\
\hline Mortality rate & Age-specific, estimated from Nunavut & & Statistics Canada [20] \\
\hline
\end{tabular}


Table 1 Model parameters, values, ranges, and sources (Continued)

Screening and Treatment Parameters

Time to diagnosis for active TB disease

Tian [27]

\begin{tabular}{|c|c|c|c|}
\hline & High & 0.5 & \\
\hline & Low & 0.64 & \\
\hline & Extrapulmonary & 0.64 & \\
\hline Time in treatment (years) & Active TB disease & 0.6 & Kivalliq surveillance data \\
\hline \multirow{3}{*}{$\begin{array}{l}\text { Probability lost to follow-up while on treatment for } \\
\text { active TB disease }\end{array}$} & & & Kivalliq surveillance data \\
\hline & Adult & 0.06 & \\
\hline & Child & 0.04 & \\
\hline Passive population screening for LTBI (per year) & & 0.004 & TAIMA TB report [34] \\
\hline $\begin{array}{l}\text { Average time to LTBI treatment initiation for cases identified } \\
\text { by population screening (months) }\end{array}$ & & 1 & Assumption \\
\hline Average time to completion of contact tracing (months) & & 2 & Tian [13] \\
\hline Time on treatment for LTBI (years) & & 0.75 & Canadian TB Standards [24] \\
\hline Probability LTBI treatment is completed & & 0.7 & Alvarez [5] \\
\hline
\end{tabular}

parameters describing baseline contact tracing and screening assumptions are found in Table 1.

We assumed that contact tracing was only done for household contacts of diagnosed index cases. Identified household contacts with LTBI (meeting age and treatment history criteria) were offered treatment, with a proportion completing treatment based on Nunavut treatment completion data (Table 1).

\section{Model calibration}

We used model calibration to estimate the number of individuals with latent, undiagnosed, or previously treated TB upon model initiation, as well as the annual number of respiratory contacts sufficient to transmit infection. To account for the fact that the risk of transmission is concentrated among close contacts (household contacts in our model), we assumed that the majority of respiratory contacts occurring between cases and their contacts occurred in the household. In our base case, we assumed that $5 \%$ of respiratory contacts sufficient for transmitting TB occurred within the community (with the remaining $95 \%$ of transmission-sufficient contacts occurring with household members). We also repeated the calibration process assuming that $1 \%$ or $15 \%$ of

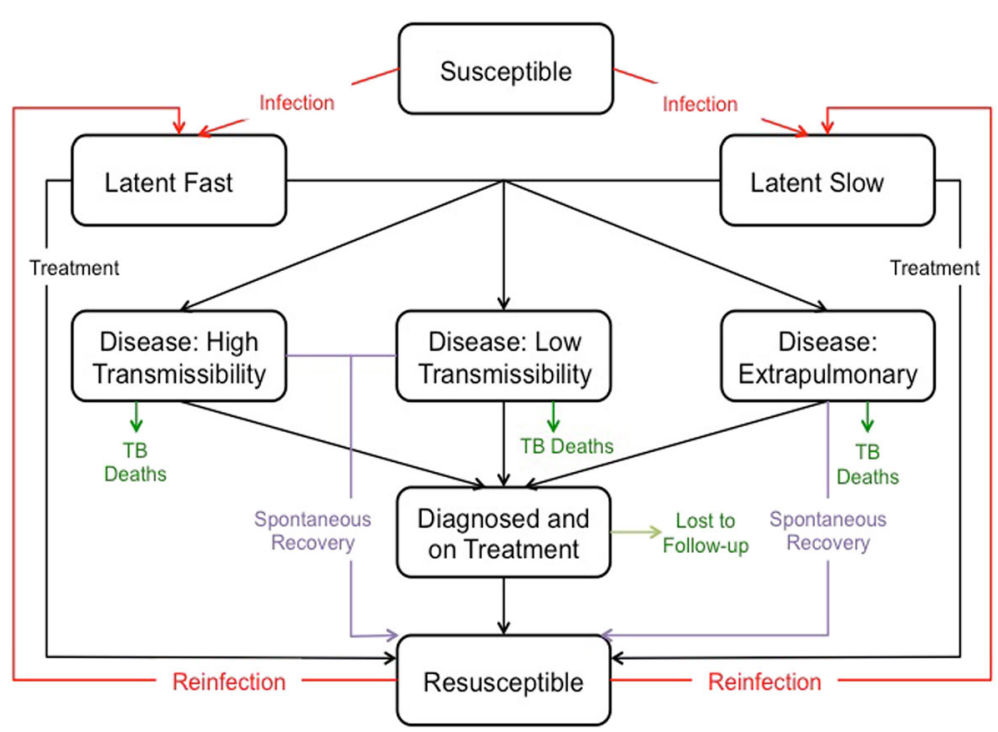

Fig. 2 Model overview. Only individuals with high or low transmissibility disease are infectious. All health states also have age-specific mortality, and the births are added to the susceptible state (not shown). The transition probabilities for certain health states differ depending on whether an individual is a child ( $<15$ years of age) or adults, as described in the text and Table 1 
respiratory contacts occurred within the community. A total of 10 best-fit parameter sets were obtained for each value of community transmission.

\section{Interventions to reduce tuberculosis burden}

We considered different interventions to reduce the burden of TB in Kivalliq (Table 2). For each intervention, changes made for that specific intervention were layered on top of the existing TB control activities that were assumed in our base case. For instance, there was a base case level of population LTBI screening in all interventions, which was increased in our population screening intervention. We included interventions that reduced TB transmission by active cases (interventions a and b), as well as those that prevented progression to active disease in LTBI cases (interventions c, d, and e):

(a) Rapid treatment of active cases: This approach relies on the timely diagnosis of individuals with active TB, with rapid initiation of treatment, such that individuals are no longer infectious to others.

(b)Increased housing to reduce overcrowding: Another way of reducing TB transmission is by decreasing the effective number of case contacts. Since the majority of TB transmission is expected to occur in households, we evaluated increasing housing availability, thereby reducing the average household size and the number of individuals potentially exposed to an infectious individual in the household setting.

Table 2 Model interventions

\begin{tabular}{|c|c|}
\hline Intervention & Details \\
\hline Base case & $\begin{array}{l}\text { - Time from active disease onset to } \\
\text { treatment: } 0.5 \text { years for pulmonary } \\
\text { high, } 0.64 \text { years for pulmonary low } \\
\text { and extrapulmonary } \\
\text { - Contact tracing time: } 60 \text { days } \\
\text { - Population screening: } 0.004 / \text { year } \\
\text { - New households: } 30 / \text { year }\end{array}$ \\
\hline Rapid treatment of active cases & $\begin{array}{l}\text { - Time from active disease onset to } \\
\text { treatment initiation reduced by half } \\
\text { ( } 0.25 \text { years for pulmonary high, } 0.32 \\
\text { years for all other) }\end{array}$ \\
\hline Rapid contact tracing $(\mathrm{CT})$ & $\begin{array}{l}\text { - Time to testing and treatment } \\
\text { initiation for household contacts } \\
\text { of diagnosed index cases reduced } \\
\text { by half ( } 30 \text { days) }\end{array}$ \\
\hline Expanded population screening & $\begin{array}{l}\text { - Rate of general population } \\
\text { screening (with appropriate } \\
\text { treatment) increased to } \\
0.01 / \text { years }\end{array}$ \\
\hline School screening & $\begin{array}{l}\text { - Screen all children aged 5, 11, } \\
\text { and } 14 \text { annually }\end{array}$ \\
\hline $\begin{array}{l}\text { Increased housing to reduce } \\
\text { overcrowding }\end{array}$ & $\begin{array}{l}\text { - Increase number of new } \\
\text { households by } 60 / \text { year }\end{array}$ \\
\hline
\end{tabular}

(c) Rapid contact tracing: Contact-tracing focuses on contacts of recently identified active cases, as these individuals are considered at high risk of infection. As screening and treatment of household contacts is already carried out as part of TB control activities, we evaluated the impact of reducing the time to carry out such investigations.

(d) Population screening: We evaluated the impact of increasing the rate of general population screening. Individuals were randomly selected from the pool of individuals in the population with no prior history of treated active or latent TB infection.

(e) School screening: Targeted screening of school-aged children has been recommended [24]. We evaluated the impact of annual screening of children aged 5, 11 , and 14 which is currently recommended in Nunavut [7].

\section{Model outcomes}

Intervention impact was evaluated by comparing the number of incident infections, LTBI diagnoses, and diagnosed active TB cases in the presence of the intervention to the base case. To account for variability in TB dynamics between model runs, comparisons of intervention impact were made within experiments; that is, for a given experiment with a best-fit parameter set estimated from the model calibration process, we compared outcomes in the presence of the different interventions to the base case. Results are presented as the median and interquartile range for the 10 experiments. We evaluated a 10-year time horizon, as this was considered relevant for public health decision-making. As an additional analysis, we considered a 25-year time horizon, given the slow progression of $\mathrm{TB}$ and the possible subsequent delay in observing changes in disease dynamics.

\section{Supplementary analyses}

We considered alternate approaches to evaluating intervention impact and to performing model calibration. A description of these approaches and results of these analyses are presented in the Additional file 1.

\section{Results \\ Model calibration}

The best-fit model realizations captured the variability in diagnosed pulmonary TB cases in Kivalliq over the 14year time period (Fig. 3). Although our base case assumed $5 \%$ of contacts occurred within the community, we present the results of calibration for all three levels of community transmission for the sake of comparison. The proportion of incident cases in community contacts ranged from less than $1 \%$ to greater than $50 \%$, depending on the assumed intensity of respiratory contacts occurring within the community (Fig. 4). For the 5\% 
A

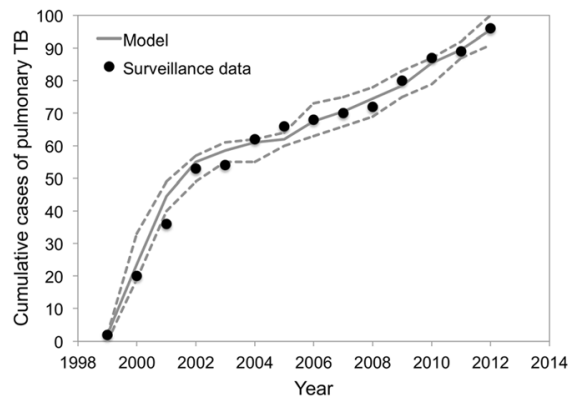

B

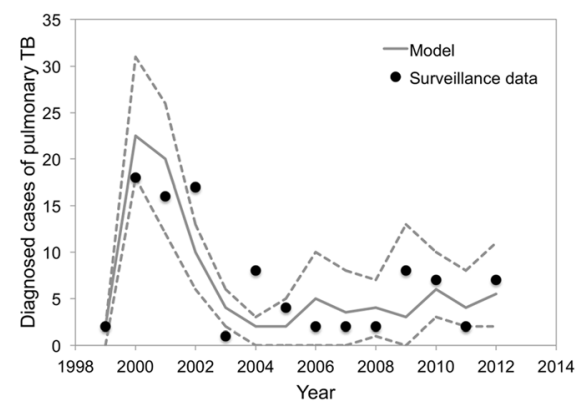

Fig. 3 Model calibration. Model-projected (a) cumulative and (b) annual cases of pulmonary TB (median: solid line; minimum/maximum: dashed lines) compared to surveillance data for the Kivalliq region of Nunavut. Results represent the 10 best-fit model realizations, assuming that 5\% of respiratory contacts sufficient for transmitting TB occur within the community. Results are similar for $1 \%$ and $15 \%$ of respiratory contacts in the community

community contacts scenario, we estimated that the contact tracing process would identify a median of $22 \%$ of household contacts with LTBI and 1.3\% of contacts with active TB infection. We observed similar estimates with lower or higher amounts of community transmission (Fig. 5).

\section{Base case scenario}

For each of the best-fit model realizations, we used the model to examine the projected dynamics of $\mathrm{TB}$ in

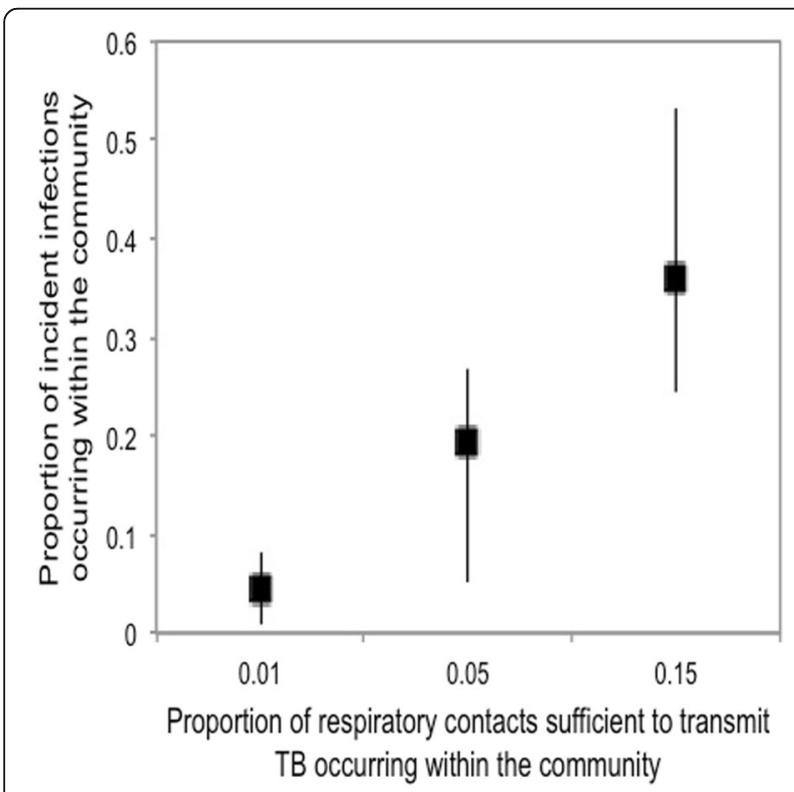

Fig. 4 Proportion of incident infections projected to occur within the community over the 14-year calibration period. Model outputs assume different levels of TB transmission within the community. The remainder of infections occur among household contacts of active TB cases. Boxes represent the median values of 10 runs, while lines span the minimum and maximum values of 10 experiments with best-fit parameters
Kivalliq over a 10-year period, in the absence of any additional interventions (Fig. 6). The ten best-fit parameter sets resulted in a high degree of variability between model runs (due to stochasticity and a small population size), with cumulative TB incidence estimates ranging from 33-369 cases over the ten year time period. Compared to the base case, decreasing the time to treatment initiation for active cases was projected to reduce the number of incident TB infections in the population and have an impact on reducing diagnosed active TB (Fig. 7). This finding was consistent across the ten best-fit parameter set experiments. In addition, expanded

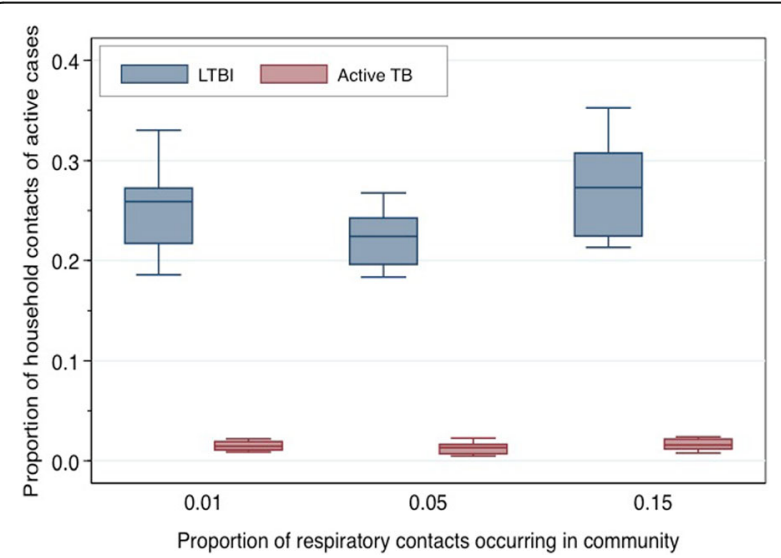

Fig. 5 Infection status of household members of active cases, identified by contact tracing. Household contacts of diagnosed cases of active TB disease had a probability of being identified and screened via the contact follow-up process, as described in the Methods. Household members may be identified as having a latent TB infection (blue boxes), or active TB disease (red boxes). Remaining screened contacts are uninfected. Results are shown for different levels of TB transmission within the community. The midpoint, lower, and upper bounds of the boxes represent the median, $25^{\text {th }}$ percentile, and $75^{\text {th }}$ percentile, respectively. Bars span 1.5 times the interquartile range 


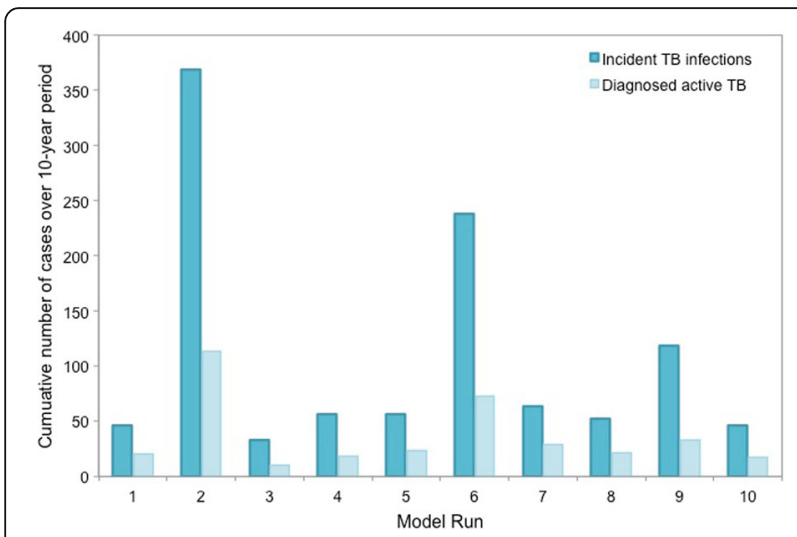

Fig. 6 Model-projected cumulative TB incidence and diagnosed cases of active TB over a 10-year period. Results are shown for the base case (i.e., no additional interventions), assuming $5 \%$ of respiratory contacts occur within the community. Each model run represents a best-fit parameter set obtained in the calibration process, as described in the Methods and Additional file 1

population screening was projected to reduce the number of incident TB infections in the population and have an impact on reducing diagnosed active TB (Fig. 7). Increased housing at the level implemented in the model trended toward reducing TB incidence, but there was variability between model runs.

As expected, compared to the base case, the two interventions that expanded LTBI screening (either at the population level or targeted to school aged children) resulted in more LTBI cases being detected (Fig. 8). However, greater LTBI detection and treatment did not necessarily translate into reduced TB burden: for the school-screening program,

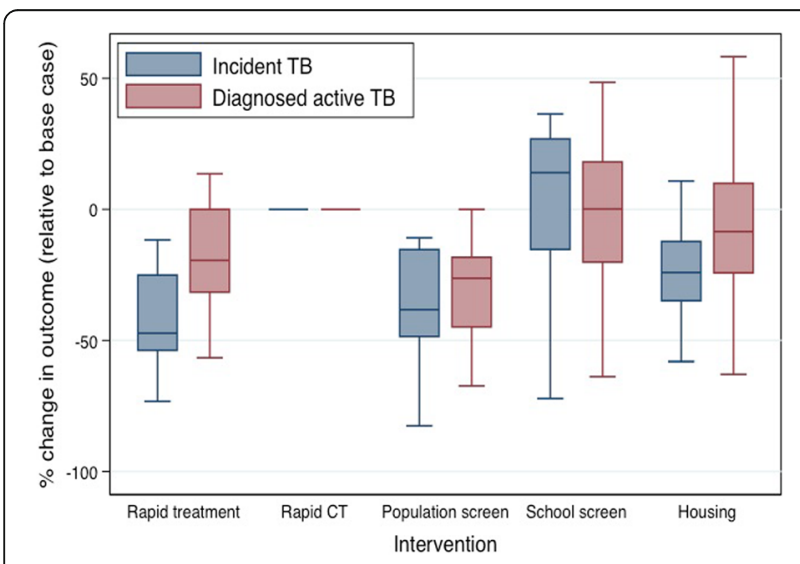

Fig. 7 Projected impact of different interventions on incident TB infections and diagnoses of active TB disease. The midpoint of boxes represents the median percent change in the outcome of interest, relative to the base case, with the upper and lower bounds representing the $25^{\text {th }}$ and $75^{\text {th }}$ percentiles of percent change, respectively and the bars indicating 1.5 times the interquartile range. Results are based on cumulative outcomes over a 10-year time horizon, assuming 5\% of transmission-sufficient respiratory contacts occur in the community. Intervention details are provided in Table 2

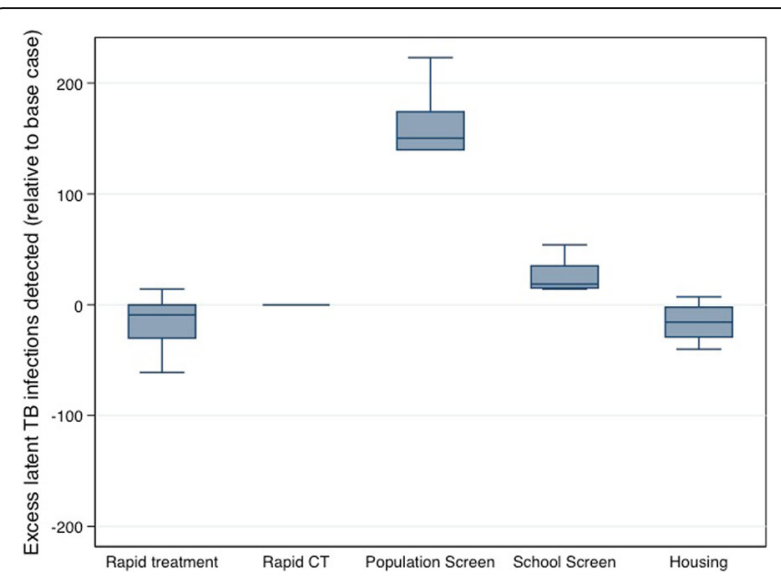

Fig. 8 Projected impact of different interventions on diagnosed latent TB infections. Results represent cumulative excess cases, relative to the base case scenario, over a 10-year period, assuming that $5 \%$ of respiratory contacts sufficient to transmit TB occur with casual community contacts, with the remaining contacts occurring with household members. The midpoint, lower, and upper bounds of the boxes represent the median, $25^{\text {th }}$ percentile, and $75^{\text {th }}$ percentile of changes in cases, respectively. Bars span 1.5 times the interquartile range. Intervention details are provided in Table 2

we did not observe a corresponding impact on incident or active TB diagnoses in the population. Reducing the time to identify, test, and where appropriate, treat contacts of infectious cases was not projected to have an impact on TB incidence or diagnoses.

\section{Sensitivity of results to model assumptions about transmission outside of households and time horizon}

Our findings were sensitive to assumptions around the relative fraction of TB transmission occurring in communities versus households (Fig. 9). When the contribution of community transmission was relatively low ( $1 \%$ of contacts sufficient to transmit TB occurred outside of the household), expected TB incidence was low (17-66 cases in the base case over a 10-year period). None of the proposed interventions were expected to have a dramatic impact on TB burden in Kivalliq, although the trend of lower incident infections and diagnosed active TB disease cases remained with the rapid treatment scenario (Fig. 9a). With higher transmission occurring outside of the household ( $15 \%$ of respiratory contacts sufficient to transmit TB occurring in the community), expected TB incidence in the base case ranged from 44 to 562 over the 10 -year period. Rapid treatment was projected to decrease TB incidence, and to a lesser extent, diagnoses of active TB disease (Fig. 9b). Expanded population screening showed a trend toward lower TB incidence and diagnoses, but there was a fair amount of variability in these findings.

Given the slow progression of TB and the possible subsequent delay in observing impact of interventions 


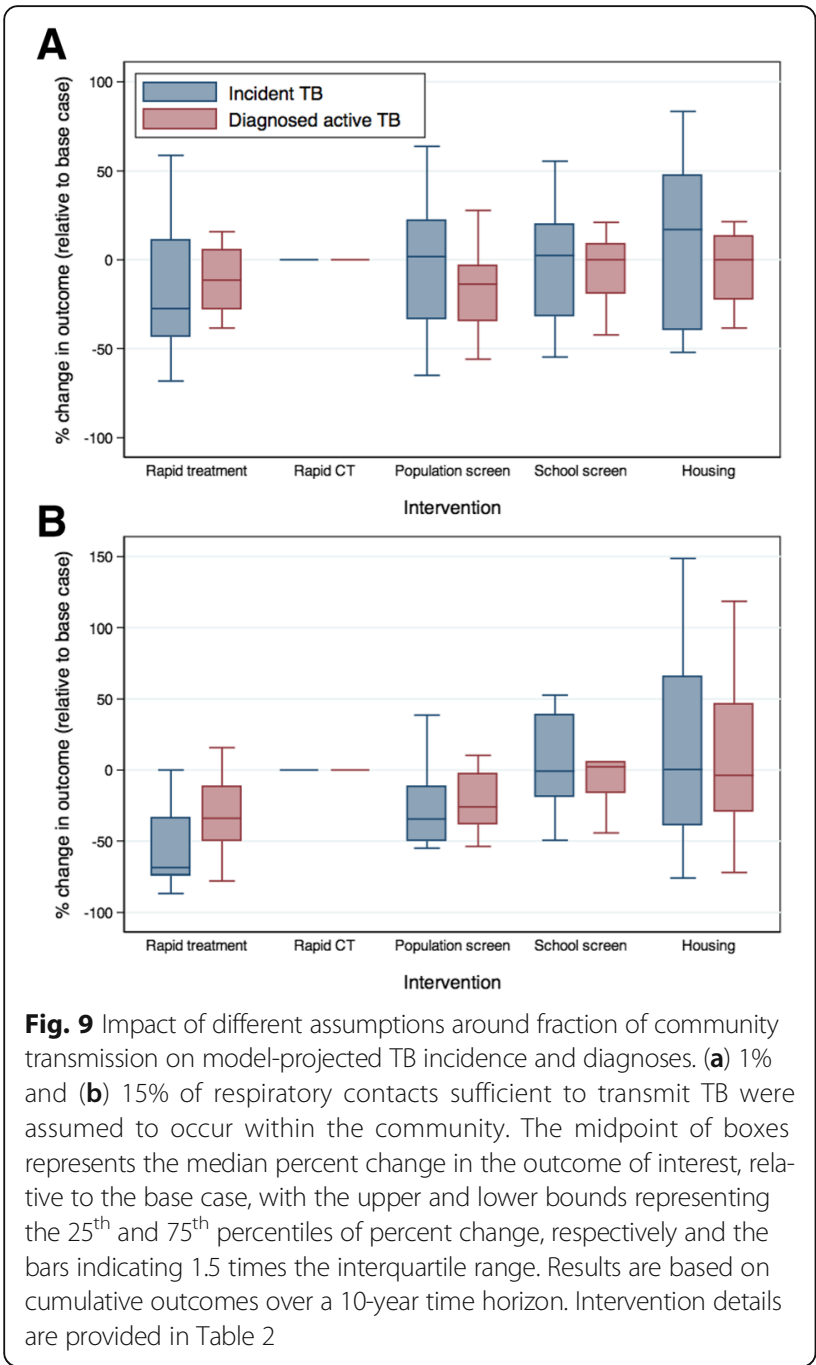

on changes in disease dynamics, we repeated our analyses using a 25-year time horizon (Fig. 10). Rapid initiation of treatment for active TB cases remained the most attractive intervention option when considering the 25-year time horizon for all scenarios (1\%, $5 \%$, and $15 \%$ community transmission). With a longer time horizon, population screening, school screening programs, and increased housing were projected to have an overall minimal effect on reducing TB burden in the population. Under the assumption of a greater number of transmission events occurring among community contacts (15\%) (Fig. 10), the rapid contact tracing intervention began to appear more attractive as an intervention, with all simulation runs resulting in a reduction in both incident and diagnosed active TB.

\section{Discussion}

We have developed an agent-based model of TB transmission in the Kivalliq Region of Nunavut. Using this model, we evaluated the potential for different
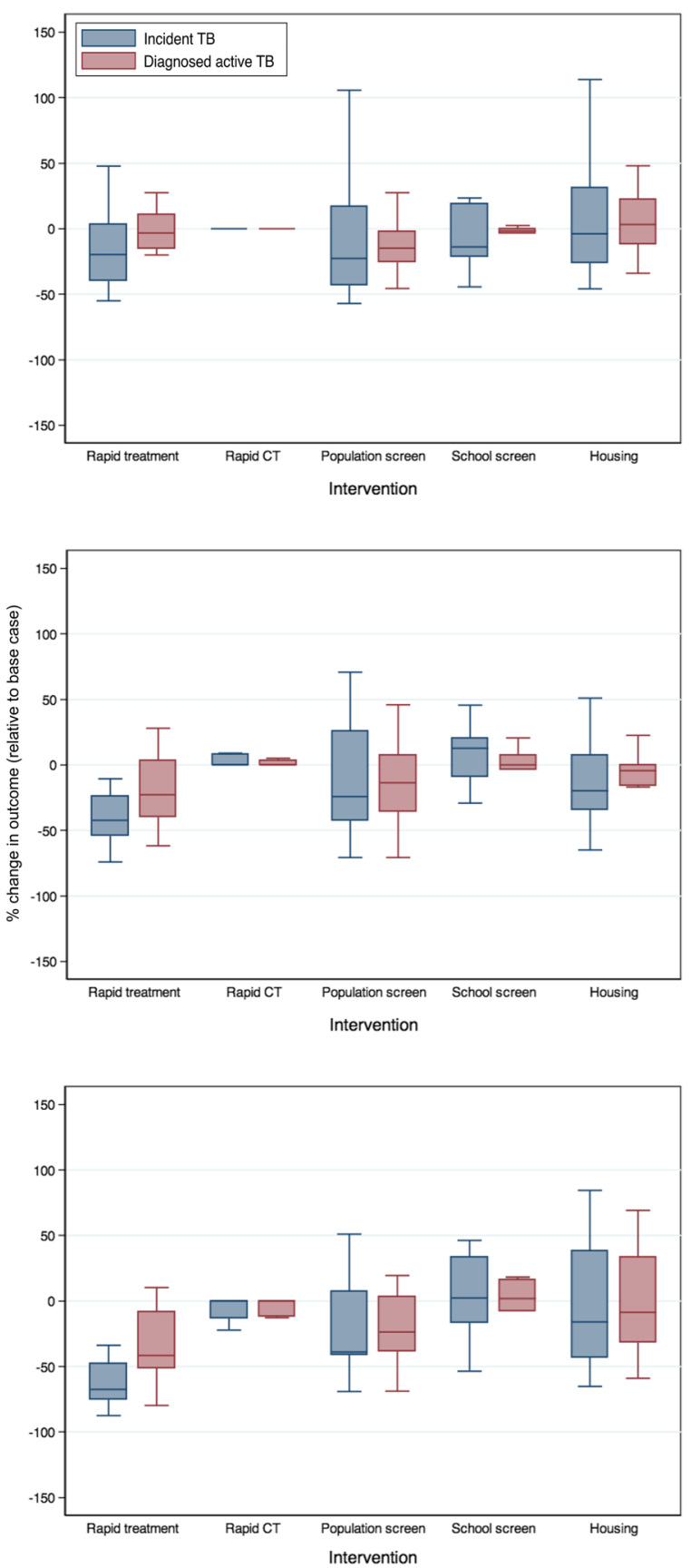

Fig. 10 Projected impact of different interventions on TB incidence and diagnoses over a 25-year period. Results are shown for 1\% (top), $5 \%$ (middle), and 15\% (bottom) of respiratory contacts occurring within the community. The midpoint of boxes represents the median percent change in the outcome of interest, relative to the base case, with the upper and lower bounds representing the $25^{\text {th }}$ and $75^{\text {th }}$ percentiles of percent change, respectively and the bars indicating 1.5 times the interquartile range. Intervention details are provided in Table 2

intervention strategies to control the spread of TB in this region. Although our results were sensitive to assumptions around the relative contribution of community transmission to TB spread, we generally found that 
reducing the time between onset of active disease and initiation of treatment was an effective means of reducing disease burden. In the short-term, expanding general population screening was also projected to reduce the burden of TB. Other potential interventions were expected to be of limited effectiveness.

Population screening and treatment of LTBI prevents the potential progression to active disease, with the downstream consequence of preventing ongoing $\mathrm{TB}$ transmission. Screening may also detect active TB cases [5]. Given that most infected individuals will not progress to active TB disease [24], many individuals need to be screened and treated to prevent a case of active disease [25] and compliance with the lengthy treatment regimen can be a challenge [24]. Despite these challenges, we found that population screening in Kivalliq was projected to reduce the burden of TB, although the effect was less pronounced when we considered a 25year time horizon.

Implementing screening programs in school-aged children was not projected to impact TB burden. As pediatric cases are less likely to have highly transmissible TB [26], it may be that finding and treating LTBI in this population is ineffective as a means of preventing disease transmission. Other targeted screening programs, focusing on population-groups considered to be at higher risk for TB infection or progression to active disease, or individuals who are at increased risk of transmitting TB to vulnerable individuals, might be expected to be of higher yield, but would require additional model complexity and data to evaluate.

We found that reducing the time to conduct contact tracing had minimal impact on disease dynamics. This finding is consistent with work by Tian et al. [27], who used a system dynamic model describing TB in Saskatchewan to demonstrate that more rapid contract tracing did not significantly impact TB incidence. A previous modeling study [11] found that follow-up of household contacts could reduce TB burden, but compared contact tracing to disease trends in the absence of any contact tracing, as opposed to investigating the role of reducing the time to perform contact tracing and initiate treatment as in the present study. Our findings suggest that the current time frame for contact tracing is adequate for detecting exposed individuals of index cases prior to their development of active infection. It is important to note that this model assumes that contact tracing is only applied to household members of active cases. It is possible that a model that included a more complex tracking of community contacts would observe a different impact of reducing contact tracing time, although, as mentioned above, the results of another mathematical modeling study suggest that expanded breadth of contact tracing has diminishing returns [27].
Household overcrowding is a recognized issue in northern communities [8]. Given the importance of household transmission for TB spread, we hypothesized that reducing the average number of individuals living in a household through the addition of households in communities would reduce TB transmission. However, we did not observe a significant impact on TB incidence when we implemented this intervention, although we did observe a trend toward lower TB incidence in our moderate community transmission scenario. It is possible that by allowing individuals to move between households (via the creation of new households), the effective number of contacts of an infectious individual actually increases. For instance, an active case might share a household with four others; if that person is then relocated to a new household with four other individuals, he has the opportunity to infect eight individuals over the course of his infection. Since contact tracing is only applied to an active case's household members at the time of diagnosis, we may be underestimating the potential for increased housing to impact TB spread. It is also possible that the impact of housing on reducing overcrowding (and downstream, transmission events) takes a longer time to manifest than that considered in this analysis. Finally, it may be that the actual number of new housing units required to significantly impact overcrowding is greater than that considered in this scenario. It should be noted that adding housing reduces average household size (and therefore the number of contacts) only. We did not model the possibility that new housing units with improved ventilation might reduce the probability of transmission per contact.

We assumed that household contacts of active cases were at greater risk of TB infection than community and casual contacts. As the degree to which community transmission contributes to TB transmission in Kivalliq is unknown, we conducted scenario analyses for differing amounts of transmission occurring in the community. The overall projected TB burden scaled with the assumed amount of community transmission, with lower levels of TB transmission expected to occur when transmission primarily occurs within the household. Based on past experience, it appears that the higher community transmission scenarios are more likely to reflect population mixing in Kivalliq. Given the importance of assumptions around the contribution of community transmission to TB dynamics, the use of molecular epidemiological techniques to better define transmission networks [28-30] might facilitate the selection of optimal disease control strategies. For example, the identification of large single-strain clusters that include both household contacts and community members would argue in favour of more community transmission, whereas the identification of sporadic strain types in the 
community, and with clusters restricted to household groups, would suggest less community-based transmission.

The failure to observe robust effects for any of the interventions in the low community transmission scenario may reflect the small absolute number of TB cases projected to occur. The median number of diagnosed cases was 20 (in the base case and in the absence of additional interventions), making it challenging to detect small or moderate differences in health outcomes upon introduction of additional interventions.

As with any model-based analysis, ours has limitations. This model includes a large number of parameters relating to the natural history of TB and treatment, many of which are subject to uncertainty [31], and also includes many simplifying assumptions. Wherever possible, we have used parameters specific for Kivalliq, Nunavut, or Canada. We have assumed that the proportion of respiratory contacts sufficient to transmit TB in the community (outside of household contacts) ranged from 1 to $15 \%$. Improved data on contact patterns between individuals would better inform our model parameterization: in particular, diary data recording specific contact patterns [32], or a detailed contact-tracing registry. Although we were able to generate model realizations that fit the available surveillance data well, the variability in the possible trajectory of TB burden over the subsequent 10 years highlights the degree of uncertainty in our model projections. We made comparisons within model realizations to account for the differences in model projections and focused on relative differences to account for this uncertainty in model projections. We have also addressed this issue in supplementary analyses presented in the Additional file 1.

\section{Conclusions}

To summarize, we have developed an agent-based model describing TB transmission in a small northern population. We have identified possible areas of TB control where increased efforts are expected to have an impact, as well as areas where focusing efforts are not expected to have as great of a payoff, in terms of reduced TB burden in the community. This model provides a platform that can be refined as we gather additional surveillance and programmatic data and can be modified to represent different communities in Canada's north. In addition to providing qualitative estimates of the relative impact of different interventions and combinations of interventions, it can be used as a tool to identify knowledge gaps [31].

\section{Additional file}

Additional file 1: Model technical appendix. (DOCX $497 \mathrm{~kb}$ )
Abbreviations

CT: Contact tracing; LTBI: Latent tuberculosis infection; TB: Tuberculosis

\section{Acknowledgements}

The authors thank Ping Yan, Tom Wong, and Susanna Ogunnaike-Cooke (Public Health Agency of Canada), and Dr. Maureen Baikie (former Medical Officer of Health for the territory of Nunavut) for providing valuable input and data.

\section{Funding}

This work was funded by the Public Health Agency of Canada (AT and AG) and by the Canada Research Chairs Program (AG). The model structure and assumptions were defined in collaboration with TB surveillance and clinical experts at both the Public Health Agency of Canada and the Nunavut

Department of Health.

\section{Availability of data and materials}

Model parameters are included in this published article. Surveillance data used for model calibration are available from the corresponding author on reasonable request.

\section{Authors' contributions}

All authors were involved in the study conception and design, analysis and interpretation of data, and drafting of the manuscript. AT built the model and analyzed output data. AG, VG, A-CB, and ER assisted with data acquisition and the critical revision of the manuscript for important intellectual content. All authors read and approved the final manuscript.

\section{Competing interests}

The authors declare that they have no competing interests.

\section{Consent for publication}

Not applicable.

\section{Ethics approval and consent to participate}

Surveillance data from the Public Health Agency of Canada and Nunavut Department of Health were provided to the research team as aggregate data and with no personal identifying features. Therefore, research ethics board (REB) approval for the use of surveillance data to inform the modeling work conducted was waived.

\section{Author details}

${ }^{1}$ Dalla Lana School of Public Health, University of Toronto, University of Toronto, Toronto, ON, Canada. ${ }^{2}$ Centre for Communicable Diseases and Infection Control, Public Health Agency of Canada, Ottawa, ON, Canada.

${ }^{3}$ Nunavut Department of Health, Iqaluit, NU, Canada. ${ }^{4}$ Department of

Population Medicine, University of Guelph, Guelph, ON, Canada. ${ }^{5}$ Harvard T.H. Chan School of Public Health, 1633 Tremont Street, Boston, MA 02120,

United States.

Received: 19 August 2016 Accepted: 23 December 2016

Published online: 13 January 2017

\section{References}

1. Halverson J, Ellis E, Gallant V, Archibald CP. Epidemiology of tuberculosis in Canada. In: Public Health Agency of Canada. Canadian Tuberculosis Standards. 7th Ed. 2013. www.phac-aspc.gc.ca/tbpc-latb/pubs/tb-canada-7/ tb-standards-tb-normes-ch1-eng.php. Accessed 5 Oct 2016.

2. Alvarez G, Orr P, Wobeser W, Cook V, Long R. Tuberculosis Prevention and Care in First Nations, Inuit and Métis Peoples. In: Public Health Agency of Canada. Canadian Tuberculosis Standards 7th Ed. 2013. p. 345-60. http://www.respiratoryguidelines.ca/tb-standards-2013. Accessed 5 Oct 2016.

3. Bourgeois A-C. Report on the epidemiology of tuberculosis in Nunavut, 1999 to 2011. Ottawa: Public Health Agency of Canada. 2013. http://www. gov.nu.ca/sites/default/files/files/Nunavut\%20Epi\%20report\%202013.pdf.

4. Public Health Agency of Canada. Tuberculosis in Canada 2012, Pre-release. 2014. http://healthycanadians.gc.ca/publications/diseases-conditionsmaladies-affections/tuberculosis-2014-tuberculose/index-eng.php. Accessed 26 Sept 2016. 
5. Alvarez GG, VanDyk DD, Aaron SD, Cameron DW, Davies N, Stephen N, et al. TAIMA (Stop) TB: The Impact of a Multifaceted TB Awareness and Door-toDoor Campaign in Residential Areas of High Risk for TB in lqaluit, Nunavut. PLoS One. 2014;9, e100975.

6. $\quad$ Orr P. Tuberculosis in Nunavut: Looking back, moving forward. Can Med Assoc J. 2013;185:287-8.

7. MacDonald N, Hébert PC, Stanbrook MB. Tuberculosis in Nunavut: a century of failure. Can Med Assoc J. 2011;183:110160.

8. Statistics Canada. An analysis of the housing needs in Nunavut: Nunavut Housing Needs Survey. 2010. http://www.gov.nu.ca/sites/default/files/files/ Analysis\%20of\%20the\%20Housing\%20Needs\%20in\%20Nunavut,\%2020092010.pdf. Accessed 16 July 2014.

9. Public Health Agency of Canada. Canadian Tuberculosis Standards, 7th Edition. Ottawa: Government of Canada; 2013.

10. Ayles H, Muyoyeta M, Du Toit E, Schaap A, Floyd S, Simwinga M, et al. Effect of household and community interventions on the burden of tuberculosis in southern Africa: The ZAMSTAR community-randomised trial. Lancet. 2013; 382:1183-94.

11. Kasaie P, Andrews JR, Kelton WD, Dowdy DW. Timing of tuberculosis transmission and the impact of household contact tracing: An agent-based simulation model. Am J Respir Crit Care Med. 2014;189:845-52.

12. Abu-Raddad $L$, Sabatelli $L$, Achterberg JT, Sugimoto JD, Longini IM, Longini Ir IM, et al. Epidemiological benefits of more-effective tuberculosis vaccines, drugs, and diagnostics. Proc Natl Acad Sci U S A. 2009;106:13980-5.

13. Tian Y. Agent-based modeling and system dynamics modeling on transmission of tuberculosis in Saskatchewan. 2012. Thesis. University of Saskatchewan. http://www.collectionscanada.gc.ca/obj/thesescanada/vol2/ SSU/TC-SSU-201110300.pdf.

14. Dowdy DW, Chaisson RE, Moulton LH, Dorman SE. The potential impact of enhanced diagnostic techniques for tuberculosis driven by HIV: a mathematical model. Aids. 2006;20:751-62.

15. Murray CJ, Salomon JA. Modeling the impact of global tuberculosis control strategies. Proc Natl Acad Sci U S A. 1998;95:13881-6.

16. CBC news. Nunavut's TB battle continues with 42 new cases this year. 2014. http://www.cbc.ca/news/canada/north/nunavut-s-tb-battle-continues-with42-new-cases-this-year-1.2672832 Accessed 30 Sept 2014.

17. Statistics Canada. Community Highlights for Keewatin Region. Cat no. 93F0053X. Community Profiles. 2001 http://www12.statcan.ca/english/ profil01/CP01/Index.cfm?Lang=E. Accessed 16 July 2014.

18. Statistics Canada. Keewatin, Nunavut (Code 6205) and Nunavut (Code 62) (table) Census Profile. 2011 Census. Stat. Canada Cat. no.98-316-XWE. 2011 http://www12.statcan.ca/census-recensement/2011/dp-pd/prof/index. cfm?Lang=E Accessed 16 July 2016

19. Statistics Canada. Age and sex, 2006 Census. Cat. No. 97-577-XIE. 2007 http://www5.statcan.gc.ca/bsolc/olc-cel/olc-cel?catno=97-551-X. Accessed 16 July 2014.

20. Statistics Canada. Life Tables, Canada, Provinces and Territories, 2007 to 2009 (84-537-X). 2013. http://www.statcan.gc.ca/pub/84-537-x/2013003/tbleng.htm 2013. Accessed 16 July 2014.

21. Nunavut Bureau of Statistics. Nunavut live births by age of mother and total fertility rate. 2013. http://www.stats.gov.nu.ca/en/Population births.aspx. 2013. Accessed 16 July 2016.

22. Long R, Schwartzman K. Pathogenesis and Transmission of Tuberculosis. In: Canadian Tuberculosis Standards. 7th edition. Public Health Agency of Canada; 2014. http://www.phac-aspc.gc.ca/tbpc-latb/pubs/tb-canada-7/tbstandards-tb-normes-ch2-eng.php. Accessed 7 Oct 2016.

23. Kitai I, Demers A-M. Pediatric Tuberculosis. Canadian Tuberculosis Standards. Public Health Agency of Canada; 2014. http://www.phac-aspc.gc.ca/tbpclatb/pubs/tb-canada-7/tb-standards-tb-normes-ch9-eng.php. Accessed 16 July 2014

24. Public Health Agency of Canada. Canadian Tuberculosis Standards, 7th Edition. 2013.

25. Rose DN. Benefits of screening for latent Mycobacterium tuberculosis infection. Arch Intern Med. 2000;160:1513-21.

26. Newton S, Brent A, Anderson S, Whittaker E, Kampmann B. Paediatric tuberculosis. Lancet Infect Dis. 2008;8:498-510.

27. Tian Y, Alawami F, Al-Azem A, Osgood N, Hoeppner V, Dutchyn C, editors. A System Dynamics model of tuberculosis diffusion with respect to contact tracing investigation. Proceedings of the 2011 Winter Simulation Conference (WSC); 2011 11-14 Dec. 2011.
28. Alexander D, Guthrie J, Pyskir D, Maki A, Kurepina N, Kreiswirth B. Mycobacterium tuberculosis in Ontario, Canada: Insights from IS6110 restriction fragment length polymorphism and mycobacterial interspersed repetitive-unit-variable-number tandem-repeat genotyping. J Clin Microbiol. 2009;47:2651-4

29. Anderson L, Tamne S, Brown T, Watson J, Mullarkey C, Zenner D. Transmission of multidrug-resistant tuberculosis in the UK: a cross-sectional molecular and epidemiological study of clustering and contact tracing. Lancet Infect Dis. 2014;14:406-15.

30. Augustynowicz-Kopec E, Jagielski T, Kozinska M, Kremer K, van Soolingen D, Bielecki J. Transmission of tuberculosis within family-households. J Infect. 2012;64:596-608.

31. Dowdy D, Dye C, Cohen T. Data needs for evidence-based decisions: a tuberculosis modeler's "wish list.". Int J Tuberc Lung Dis. 2013;17:866-77.

32. Mossong J, Hens N, Jit M, Beutels P, Auranen K, Mikolajczyk R, et al. Social contacts and mixing patterns relevant to the spread of infectious diseases. Plos Med. 2008:5:381-91.

33. Vynnycky E, Fine PE. The natural history of tuberculosis: the implications of age-dependent risks of disease and the role of reinfection. Epidemiol Infect. 1997;119:183-201.

34. Alvarez GG, Taima TB. Progress Report. 2012

\section{Submit your next manuscript to BioMed Central and we will help you at every step:}

- We accept pre-submission inquiries

- Our selector tool helps you to find the most relevant journal

- We provide round the clock customer support

- Convenient online submission

- Thorough peer review

- Inclusion in PubMed and all major indexing services

- Maximum visibility for your research

Submit your manuscript at www.biomedcentral.com/submit
) Biomed Central 